\title{
Incomplete Pairwise Comparison Matrices and Weighting Methods*
}

\author{
László Csató†- Lajos Rónyai ${ }^{\ddagger}$
}

August 28, 2015

\begin{abstract}
A special class of preferences, given by a directed acyclic graph, is considered. They are represented by incomplete pairwise comparison matrices as only partial information is available: for some pairs no comparison is given in the graph. A weighting method satisfies the linear order preservation property if it always results in a ranking such that an alternative directly preferred to another does not have a lower rank. We study whether two procedures, the Eigenvector Method and the Logarithmic Least Squares Method meet this axiom. Both weighting methods break linear order preservation, moreover, the ranking according to the Eigenvector Method depends on the incomplete pairwise comparison representation chosen.
\end{abstract}

Keywords: Directed acyclic graph; incomplete pairwise comparison matrix; Eigenvector Method; Logarithmic Least Squares Method

JEL classification number: $\mathrm{C} 44$

AMS classification number: 15A06, 90B50, 91B08

\section{Introduction}

Pairwise comparisons are widely used in multi-attribute decision making since Saaty published the AHP method [12]. It is assumed that decision makers give a numerical answer to the question 'How many times is the $i$ th alternative more important/better/favorable than the $j$ th?', which are incorporated into a matrix with an appropriate size.

Let $\mathbb{R}_{+}^{n}$ denote the positive orthant of the $n$-dimensional Euclidean space and $\mathbb{R}_{+}^{n \times n}$ denote the set of positive matrices of size $n \times n$.

Definition 1. Pairwise comparison matrix: Matrix $\mathbf{A}=\left[a_{i j}\right] \in \mathbb{R}_{+}^{n \times n}$ is a pairwise comparison matrix if $a_{j i}=1 / a_{i j}$ for all $i, j=1,2, \ldots, n$.

* We are grateful to Sándor Bozóki for reading the manuscript and for useful advices.

The research was supported by OTKA grants K 111797 and NK 105645.

$\dagger$ Department of Operations Research and Actuarial Sciences, Corvinus University of Budapest (BCE) and MTA-BCE "Lendület" Strategic Interactions Research Group, Budapest, Hungary

e-mail: laszlo.csato@uni-corvinus.hu

$\ddagger$ Informatics Laboratory, Institute for Computer Science and Control, Hungarian Academy of Sciences (MTA SZTAKI) and Budapest University of Technology and Economics (BME), Budapest, Hungary e-mail: ronyai.lajos@sztaki.mta.hu 
The final aim of the use of pairwise comparisons is to determine a weight vector $\mathbf{w}=\left[w_{i}\right] \in \mathbb{R}_{+}^{n}$ for the alternatives such that $w_{i} / w_{j}$ somehow approximates $a_{i j}$.

Definition 2. Consistency: Pairwise comparison matrix $\mathbf{A}=\left[a_{i j}\right]$ is consistent if $a_{i k}=$ $a_{i j} a_{j k}$ for all $i, j, k=1,2, \ldots, n$.

Every consistent pairwise comparison matrix can be associated to a weight vector $\mathbf{w}$ where $a_{i j}=w_{i} / w_{j}$ for all $i, j=1,2, \ldots, n$. Vector $\mathbf{w}$ is unique up to multiplication by positive scalars.

Pairwise comparison matrices provided by decision makers are usually do not meet the consistency condition. In other words, they are inconsistent. Then the real weight vector w can only be estimated on the basis of the inconsistent pairwise comparison matrix. A number of weighting methods is proposed for this purpose.

Saaty [12] used the Perron theorem [11]: a positive matrix has a dominant eigenvalue with multiplicity one and an associated strictly positive (right) eigenvector.

Definition 3. Eigenvector Method $(E M)$ [12]: $E M$ gives the weight vector $\mathbf{w}^{E M}(\mathbf{A}) \in$ $\mathbb{R}_{+}^{n}$ for any pairwise comparison matrix $\mathbf{A}$ such that

$$
\mathbf{A w}^{E M}(\mathbf{A})=\lambda_{\max } \mathbf{w}^{E M}(\mathbf{A}),
$$

where $\lambda_{\max }$ denotes the maximal eigenvalue, also known as Perron eigenvalue, of matrix A.

Distance-minimization techniques minimize the function $\sum_{i} \sum_{j} d\left(a_{i j}, w_{i} / w_{j}\right)$, where $d\left(a_{i j}, w_{i} / w_{j}\right)$ is some sort of a distance of $a_{i j}$ from its approximation $w_{i} / w_{j}$. The following is an important example with $d\left(a_{i j}, w_{i} / w_{j}\right)=\left[\log a_{i j}-\log \left(w_{i} / w_{j}\right)\right]^{2}$.

Definition 4. Logarithmic Least Squares Method (LLSM) [3; 4; 5]: LLSM gives the weight vector $\mathbf{w}^{L L S M}(\mathbf{A}) \in \mathbb{R}_{+}^{n}$ for any pairwise comparison matrix $\mathbf{A}$ as the optimal solution of the problem:

$$
\min _{\mathbf{w} \in \mathbb{R}_{+}^{n}, \sum_{i=1}^{n} w_{i}=1} \sum_{i=1}^{n} \sum_{j=1}^{n}\left[\log a_{i j}-\log \left(\frac{w_{i}}{w_{j}}\right)\right]^{2} .
$$

It may also happen that some pairwise comparisons are unknown due to the lack of available data, uncertain evaluations, or other problems. Incomplete pairwise comparison matrices were introduced in Harker [7].

Definition 5. Incomplete pairwise comparison matrix: Matrix $\mathbf{A}=\left[a_{i j}\right]$ of size $n \times n$ is an incomplete pairwise comparison matrix if $a_{i i}=1$ for all $i=1,2, \ldots, n$, and for all $i \neq j, a_{j i}=1 / a_{i j} \in \mathbb{R}_{+}$or both $a_{i j}$ and $a_{j i}$ are missing.

Notation 1. Missing elements of pairwise comparison matrices are denoted by $*$.

Example 1. The following pairwise comparison matrix of size $4 \times 4$ is incomplete:

$$
\mathbf{A}=\left(\begin{array}{cccc}
1 & * & a_{13} & a_{14} \\
* & 1 & a_{23} & * \\
1 / a_{13} & 1 / a_{23} & 1 & a_{34} \\
1 / a_{14} & * & 1 / a_{34} & 1
\end{array}\right)
$$


Generalization of $E M$ to incomplete pairwise comparison matrices requires some comment on measuring inconsistency. Saaty [12] defined the $C R$ index as

$$
C R(\mathbf{A})=\frac{\left(\lambda_{\max }(\mathbf{A})-n\right) /(n-1)}{\left(\overline{\lambda_{\max }^{n \times n}}-n\right) /(n-1)}=\frac{\lambda_{\max }(\mathbf{A})-n}{\overline{\lambda_{\max }^{n \times n}}-n},
$$

where $\overline{\lambda_{\max }^{n \times n}}$ denotes the average value of the maximal eigenvalue of randomly generated pairwise comparison matrices of size $n \times n$ such that each element $a_{i j}, i<j$ is chosen from the set $\{1 / 9 ; 1 / 8 ; \ldots ; 1 / 2 ; 1 ; 2 ; \ldots ; 8 ; 9\}$ with equal probability. $C R(\mathbf{A})$ is a positive linear transformation of $\lambda_{\max }(\mathbf{A})$. $C R(\mathbf{A}) \geq 0$ and $C R(\mathbf{A})=0$ if and only if $\mathbf{A}$ is consistent. Saaty recommended the rule of acceptability $C R<0.1$.

The idea that larger $\lambda_{\max }$ indicates higher $(C R)$ inconsistency led $[14 ; 13]$ to introduce variables for missing elements, arranged in vector $\mathbf{x}$ and consider the eigenvalue optimization problem

$$
\min _{\mathbf{x}>0} \lambda_{\max }(\mathbf{A}(\mathbf{x}))
$$

in order to find a completion that minimizes the maximal eigenvalue, or, equivalently, $C R$.

Extension of distance-based weighting methods to the incomplete case seems to be straightforward: when calculating the optimal weights, only the known terms are considered in the objective function [10; 2].

Bozóki et al. [2] discuss the question of uniqueness of the optimal solution for $E M$ and $L L S M$ in the incomplete case, solve the $L L S M$ problem 1 and propose an algorithm for finding the best completion of an incomplete pairwise comparison matrix according to $E M$. We will use their results extensively.

This paper investigates a special class of preferences described by incomplete pairwise comparison matrices (Section 2), for which some natural rankings of the alternatives exist. Section 3 reveals that $L L S M$ does not result in one of these orders. Section 4 presents that $E M$ does not meet the required condition either. Moreover, the ranking depends on the representation chosen. These are the main results of our paper. Finally, in Section 5 , we pose some related questions.

\section{Linear order preservation}

Sometimes the decision maker can only provide an ordinal information such as the $i$ th alternative is preferred to the $j$ th [8; 6]. In this model, incomplete pairs (missing comparisons) are allowed but draws are excluded: when the $i$ th and the $j$ th alternatives have been compared, the $i$ th or the $j$ th is preferred to the other.

Definition 6. Ordinal pairwise comparison matrix: Incomplete pairwise comparison matrix $\mathbf{A}=\left[a_{i j}\right]$ of size $n \times n$ is an ordinal pairwise comparison matrix if $a_{i i}=1$ for all $i=1,2, \ldots, n$, and for all $i \neq j, a_{i j} \in\{b ; 1 / b\}$ or both $a_{i j}$ and $a_{j i}$ are missing. A real number $b>1$ is an arbitrarily fixed.

Note that the value $b>1$ corresponds to the (strict) preference relation between the alternatives.

Ordinal pairwise comparison matrices can be represented by directed graphs. Let A be an ordinal pairwise comparison matrix of size $n \times n$. Then $G:=(V, E)$ where

\footnotetext{
${ }^{1}$ See also Kaiser and Serlin [9].
} 
$V=\{1,2, \ldots, n\}$, the vertices correspond to the alternatives, and $E=\left\{e(i, j): a_{i j}=\right.$ $b, i \neq j\}$, there is a directed edge from vertex $i$ to vertex $j$ if and only if the $i$ th alternative is preferred to the $j$ th. The directed graph associated to an ordinal pairwise comparison matrix $\mathbf{A}$ is independent of the value $b>1$.

Note that different choice of the parameter $b>1$ is equivalent to taking a corresponding element-wise (positive) power of $\mathbf{A}$. In other words, the associated directed graph is the same for every $\mathbf{A}^{(h)}=\left[a_{i j}^{h}\right], h>0$.

Definition 7. Weak connectedness: Let $\mathbf{A}=\left[a_{i j}\right]$ be an ordinal pairwise comparison matrix of size $n \times n$. The directed graph associated to $\mathbf{A}$ is weakly connected if for all $k, \ell=1,2, \ldots, n$, there exists a sequence of alternatives $k=m_{0}, m_{1}, \ldots, m_{t-1}, m_{t}=\ell$ such that $a_{m_{s-1} m_{s}}$ is known for all $s=1,2 \ldots, t$.

In an ordinal pairwise comparison matrix represented by a weakly connected directed graph, all alternatives are compared directly or indirectly (i.e. through other alternatives).

Definition 8. Existence of a linear order of the alternatives: Let $\mathbf{A}=\left[a_{i j}\right]$ be an ordinal pairwise comparison matrix of size $n \times n$. There exists a linear order of the alternatives if there is a permutation $\sigma:\{1 ; 2 ; \ldots ; n\} \rightarrow\{1 ; 2 ; \ldots ; n\}$ on the set of alternatives such that $\mathbf{C}=\left[c_{i j}\right]$ is the permuted ordinal pairwise comparison matrix given by $c_{i j}=a_{\sigma(i) \sigma(j)}$ for all $i, j=1,2, \ldots, n$ and $c_{i j}=b$ if $i<j$ and $c_{i j}$ is known.

Existence of a linear order of the alternatives means that the ordinal pairwise comparison matrix can be permuted such that every known value above the diagonal is $b>1$. Regarding the directed graph representation, it is equivalent to acyclicity.

The following condition concerns the weighting methods for ordinal pairwise comparison matrices. A similar requirement, called Condition of Order Preservation (COP), was introduced by Bana e Costa and Vansnick [1]. However, it is defined on complete pairwise comparison matrices and takes into account the intensity of preferences.

Definition 9. Linear order preservation $(L O P)$ : Let $\mathbf{A}=\left[a_{i j}\right]$ be an ordinal pairwise comparison matrix of size $n \times n$ such that there exists a linear order of the alternatives. It can be assumed without loss of generality that $a_{i j}=b$ if $i<j$ and $a_{i j}$ is known. A weighting method associating a vector $\mathbf{w}(\mathbf{A}) \in \mathbb{R}_{+}^{n}$ to $\mathbf{A}$ satisfies linear order preservation if $w_{i}(\mathbf{A}) \geq w_{j}(\mathbf{A})$ for all $i<j$ such that $a_{i j}$ is known $\left(a_{i j}=b\right)$.

In an ordinal pairwise comparison matrix exhibiting a linear order of the alternatives, there exist some 'natural rankings'. Linear order preservation requires that the ranking according to the weighting method examined always corresponds to one of them.

Note that a weighting method associating the same weight for each alternative meets the property $L O P$.

\section{Linear order preservation and the Logarithmic Least Squares Method}

In this section it will be scrutinized whether $L L S M$ satisfies the property $L O P$. Notation 2. $\mathbf{y}(\mathbf{A}) \in \mathbb{R}^{n}$ is given by $y_{i}(\mathbf{A})=\log w_{i}^{L L S M}(\mathbf{A})$ for all $i=1,2, \ldots, n$. 
Proposition 1. Let $\mathbf{A}=\left[a_{i j}\right]$ be an ordinal pairwise comparison matrix of size $n \times n$. Vector $\mathbf{w}^{L L S M}(\mathbf{A})$ is unique if and only if the directed graph associated to $\mathbf{A}$ is weakly connected. Then the ranking of alternatives is independent of the value of $b>1$, that is,

$$
w_{i}^{L L S M}(\mathbf{A}) \geq w_{j}^{L L S M}(\mathbf{A}) \Leftrightarrow w_{i}^{L L S M}\left(\mathbf{A}^{(h)}\right) \geq w_{j}^{L L S M}\left(\mathbf{A}^{(h)}\right)
$$

for all $i, j=1,2, \ldots, n$ and $h>0$, where $\mathbf{A}^{(h)}=\left[a_{i j}^{h}\right]$.

Proof. The necessary and sufficient condition for uniqueness is given by Bozóki et al. [2, Theorem 4].

$$
\mathbf{y}(\mathbf{A})=\mathbf{D}(\mathbf{A}) \mathbf{r}(\mathbf{A}) \text { where } \mathbf{r}(\mathbf{A})=\left[\sum_{j: a_{i j} \text { is known }} \log a_{i j}\right] \in \mathbb{R}^{n} \text {, so } \mathbf{r}\left(\mathbf{A}^{(h)}\right)=h \mathbf{r}(\mathbf{A}) \text { and }
$$

$\mathbf{D}(\mathbf{A})$ depends only on the positions of known comparisons but is not affected by their values [2, Remark 3]. Therefore $\mathbf{y}\left(\mathbf{A}^{(h)}\right)=h \mathbf{y}(\mathbf{A})$, which proves Proposition 1.

Since linear order preservation is based on the directed acyclic graph representation of an ordinal pairwise comparison matrix, Proposition 1 states that it is meaningful to question whether $L L S M$ satisfies $L O P$.

Corollary 1. It does not depend on the choice of $b>1$ whether LLSM satisfies LOP or not. In other words, LLSM gives the same ranking for every ordinal pairwise comparison matrix associated to a given directed acyclic graph.

Intuition appears to suggest that LLSM satisfies LOP. The first of our main results contradicts this expectation.

Theorem 1. LLSM may violate LOP.

Proof. It is provided by Example 2 .

Figure 1: The directed acyclic graph of Example 2

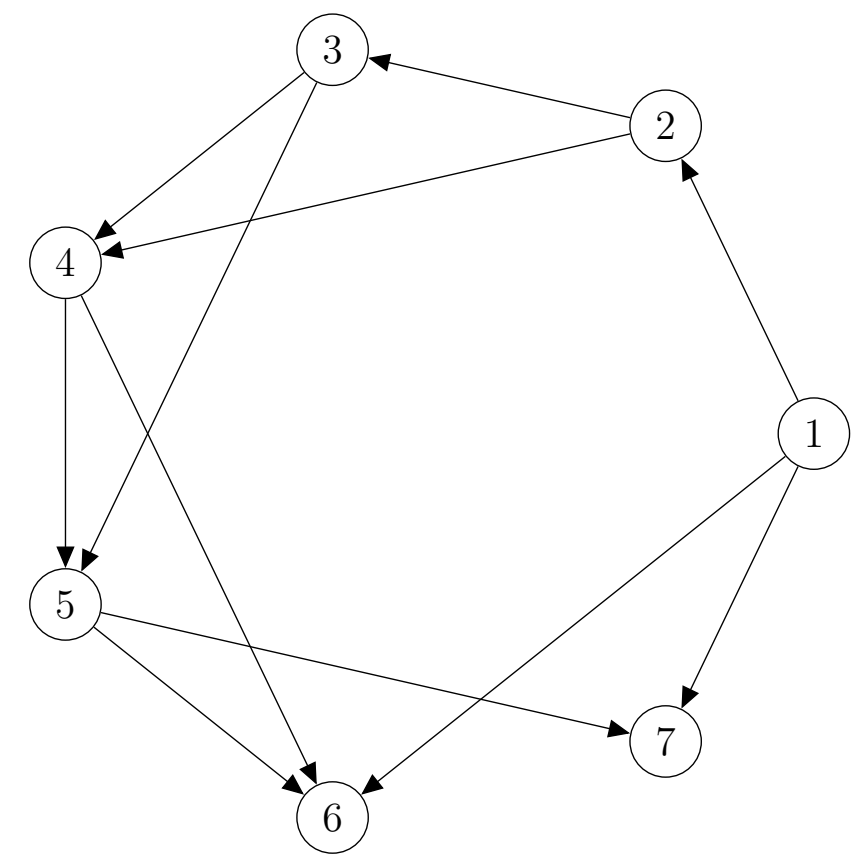


Example 2. Consider the directed acyclic graph on Figure 1. The associated ordinal pairwise comparison matrix $\mathbf{A}$ is as follows:

$$
\mathbf{A}=\left(\begin{array}{ccccccc}
1 & b & * & * & * & b & b \\
1 / b & 1 & b & b & * & * & * \\
* & 1 / b & 1 & b & b & * & * \\
* & 1 / b & 1 / b & 1 & b & b & * \\
* & * & 1 / b & 1 / b & 1 & b & b \\
1 / b & * & * & 1 / b & 1 / b & 1 & * \\
1 / b & * & * & * & 1 / b & * & 1
\end{array}\right)
$$

where $b>1$.

In Example 2, property $L O P$ is satisfied if $w_{1}(\mathbf{A}) \geq w_{2}(\mathbf{A}), w_{1}(\mathbf{A}) \geq w_{6}(\mathbf{A}), w_{1}(\mathbf{A}) \geq$ $w_{7}(\mathbf{A})$ as well as $w_{i}(\mathbf{A}) \geq w_{i+1}(\mathbf{A})$ and $w_{i}(\mathbf{A}) \geq w_{i+2}(\mathbf{A})$ for all $i=2,3,4,5$.

However, $L L S M$ results in

$$
\mathbf{y}(\mathbf{A})=\left[\begin{array}{lllllll}
34 & 36 & 24 & 1 & -14 & -42 & -39
\end{array}\right]^{\top} \log b / 49
$$

namely, $w_{1}^{L L S M}(\mathbf{A})<w_{2}^{L L S M}(\mathbf{A})$, in contradiction with preservation of linear order.

Remark 1. Example 2 is minimal regarding the number of alternatives (7) and among them, with respect to the number of known comparisons (11) 2 However, there exist more than ten examples with 7 alternatives, and some of them contain only 11 known comparisons.

Remark 2. There exist some examples to Theorem 1 with 8 alternatives and 10 known comparisons. Two of them are presented in Example 3 .

Figure 2: The directed acyclic graphs of Example 3

(a) Graph of matrix $\mathbf{A}$

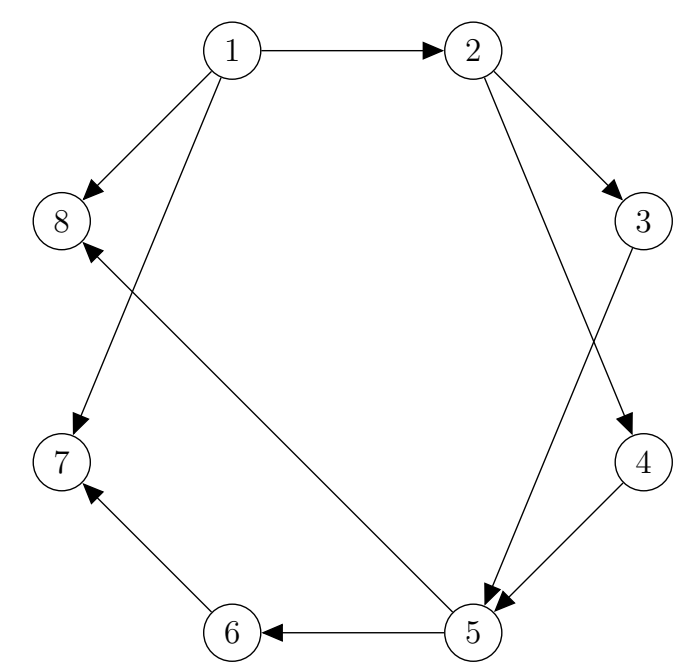

(b) Graph of matrix $\mathbf{A}^{\prime}$

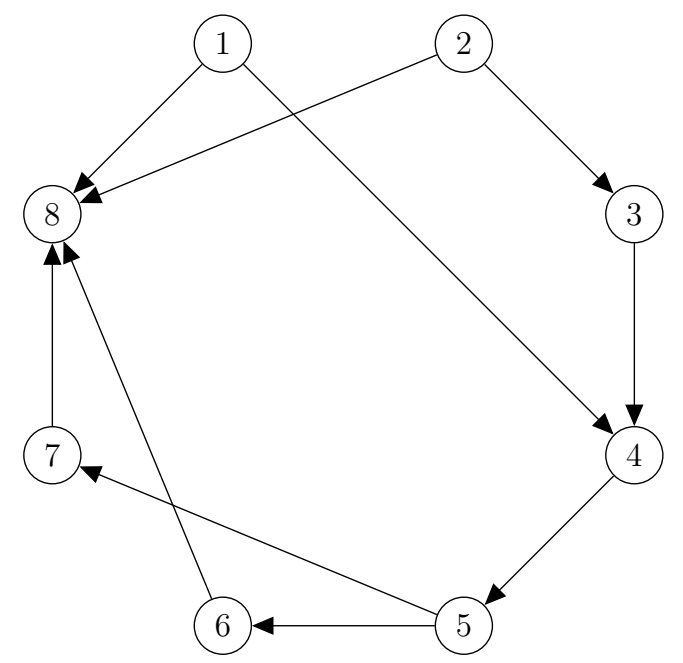

\footnotetext{
${ }^{2}$ It can be verified by brute force, examining all ordinal pairwise comparison matrices up to size $6 \times 6$. It is possible because of Corollary 1, which implies that comparisons above the diagonal may have essentially two 'values', known or missing. There exist $2^{15}=32768$ acyclic directed graphs of size $6 \times 6$.
} 
Example 3. Consider the directed acyclic graphs on Figure 2 and the associated ordinal pairwise comparison matrices $\mathbf{A}$ and $\mathbf{A}^{\prime}$. LLSM gives

$$
\begin{gathered}
\mathbf{y}(\mathbf{A})=\left[\begin{array}{llllllll}
95 & 103 & 43 & 43 & -17 & -65 & -113 & -89
\end{array}\right]^{\top} \log b / 128, \text { and } \\
\mathbf{y}\left(\mathbf{A}^{\prime}\right)=\left[\begin{array}{llllllll}
71 & 95 & 47 & -1 & 7 & -53 & -53 & -113
\end{array}\right]^{\top} \log b / 128
\end{gathered}
$$

where $w_{1}^{L L S M}(\mathbf{A})<w_{2}^{L L S M}(\mathbf{A})$ and $w_{4}^{L L S M}\left(\mathbf{A}^{\prime}\right)<w_{5}^{L L S M}\left(\mathbf{A}^{\prime}\right)$, in contradiction with preservation of linear order.

The violation of $L O P$ can be arbitrarily 'strong', regarding the difference of the weights of the alternatives involved.

Theorem 2. For every $K \in \mathbb{R}_{+}$there exists an ordinal pairwise comparison matrix $\mathbf{A}=\left[a_{i j}\right]$ exhibiting a linear order of the alternatives with a given $b>1$ such that $a_{i j}$ is known and $w_{i}^{L L S M}(\mathbf{A})-w_{j}^{L L S M}(\mathbf{A}) \leq-K$ for some $i<j$.

Proof. It is provided by Example 4 for any $k \geq 2$.

Figure 3: The directed acyclic graph of Example 4

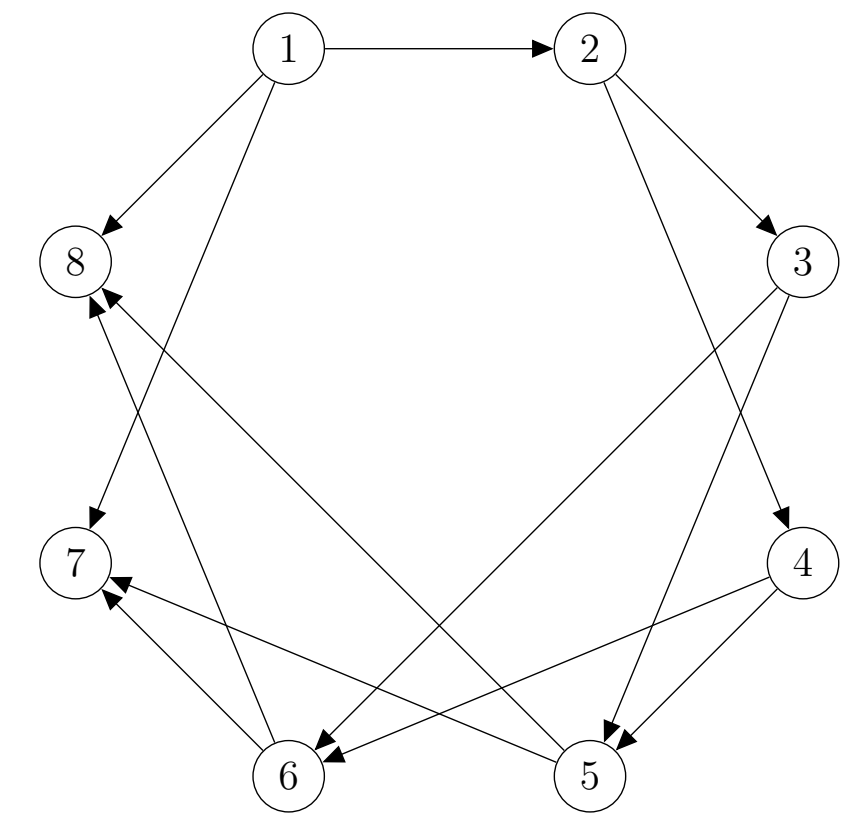

Notation 3. Let $S$ and $T$ be two sets of nodes in a directed acyclic graph. $S \rightarrow T$ if and only if there exists an edge from every vertex $i \in S$ to every vertex $j \in T$.

Example 4. Consider the family of directed acyclic graphs with $n=k m+2$ vertices where $C_{i}=\{(i-1) k+3,(i-1) k+4, \ldots, i k+2\}$ for all $i=1,2, \ldots m$ (so $C_{i}$ has $k$ elements) such that the edges are given by $\{1\} \rightarrow\{2\},\{1\} \rightarrow C_{m},\{2\} \rightarrow C_{1}$ and $C_{i} \rightarrow C_{i+1}$ for all $i=1,2, \ldots m-1$. Figure 3 shows a member of this family if $k=2$ and $m=3$, that is, $C_{1}=\{3,4\}, C_{1}=\{5,6\}$ and $C_{3}=\{7,8\}$.

The directed graphs of Example 4 are weakly connected for any pair of $k$ and $m$. $L L S M$ weights can be obtained as the solution of the following system of linear equations 
(note that alternatives of $C_{i}$ have the same weight $y_{C_{i}}$ since the $L L S M$ weight vector is unique and alternatives of $C_{i}$ are symmetric):

$$
\begin{aligned}
(k+1) y_{1}-y_{2}-k y_{C_{m}} & =k+1 ; \quad(\text { for the } 1 \text { st alternative }) \\
(k+1) y_{2}-y_{1}-k y_{C_{1}} & =k-1 ; \quad(\text { for the 2nd alternative) } \\
(k+1) y_{C_{1}}-y_{2}-k y_{C_{2}} & =k-1 ; \quad\left(\text { for alternatives in } C_{1}\right) \\
2 k y_{C_{i}}-k y_{C_{i-1}}-k y_{C_{i+1}} & =0 ; \quad\left(\text { for alternatives in } C_{i}, i=2,3, \ldots m-1\right) \\
(k+1) y_{C_{m}}-y_{1}-k y_{C_{m-1}} & =-(k+1) . \quad\left(\text { for alternatives in } C_{m}\right)
\end{aligned}
$$

For instance, the 2nd alternative has $k+1$ known comparisons: with the first, and with all $k$ alternatives of $C_{1}$, and the 2 nd is preferred in the latter $k$ of these. The derivation can be found in Bozóki et al. [2].

Subtract (2) from (11) in order to get

$$
(k+2)\left(y_{1}-y_{2}\right)-k\left(y_{C_{m}}-y_{C_{1}}\right)=2 .
$$

The difference of equations (3) and (5) gives,

$$
\left(y_{C_{1}}-y_{C_{m}}\right)-\left(y_{2}-y_{1}\right)-k\left(y_{C_{2}}-y_{C_{1}}\right)-k\left(y_{C_{m}}-y_{C_{m-1}}\right)=2 k .
$$

It follows from equations (44) that

$$
y_{C_{2}}-y_{C_{1}}=y_{C_{3}}-y_{C_{2}}=\cdots=y_{C_{m}}-y_{C_{m-1}}=\frac{y_{C_{m}}-y_{C_{1}}}{m-1} .
$$

Equations (77) and (8) lead to

$$
\left(y_{C_{1}}-y_{C_{m}}\right)-\left(y_{2}-y_{1}\right)+\frac{2 k}{m-1}\left(y_{C_{1}}-y_{C_{m}}\right)=2 k
$$

which results in

$$
y_{C_{1}}-y_{C_{m}}=\frac{m-1}{2 k+m-1}\left[2 k+\left(y_{2}-y_{1}\right)\right] .
$$

Substituting (9) into (6) gives

$$
(k+2)\left(y_{1}-y_{2}\right)=2-k\left(y_{C_{1}}-y_{C_{m}}\right)=2-\frac{k(m-1)}{2 k+m-1}\left[2 k+\left(y_{2}-y_{1}\right)\right] .
$$

After some calculation we infer

$$
\begin{aligned}
(k+2)(2 k+m-1)\left(y_{1}-y_{2}\right) & =4 k+2 m-2-2 k^{2}(m-1)-k(m-1)\left(y_{2}-y_{1}\right) ; \\
\left(2 k^{2}+4 k+2 m-2\right)\left(y_{1}-y_{2}\right) & =-2 k^{2}(m-1)+4 k+2 m-2 .
\end{aligned}
$$

It means that

$$
y_{1}-y_{2}=\frac{-k^{2}(m-1)+2 k+m-1}{k^{2}+2 k+m-1},
$$

so $\lim _{m \rightarrow \infty}\left(y_{1}-y_{2}\right)=-\infty$ for any $k \geq 2$. Hence $w_{2}^{L L S M}-w_{1}^{L L S M}$ can be arbitrarily large independent of $b .3$

Remark 3. In Example 4, $w_{1}^{L L S M}-w_{2}^{L L S M}>0$ if $k=2$ and $m=2$ (so there are 6 alternatives), but $w_{1}^{L L S M}-w_{2}^{L L S M}<0$ if $k=3$ and $m=2$ or $k=2$ and $m=3$ (so there are 8 alternatives with 13 and 16 known comparisons, respectively). This family of directed acyclic graphs does not give an example with 7 alternatives as Example 2 does.

\footnotetext{
${ }^{3}$ It is trivial if $b$ can vary as $\log b$ may also be arbitrarily large.
} 


\section{Linear order preservation and the Eigenvector Method}

In this section we examine whether $E M$ satisfies the property $L O P$ or not.

Proposition 2. Let $\mathbf{A}=\left[a_{i j}\right]$ be an ordinal pairwise comparison matrix. Vector $\mathbf{w}^{E M}(\mathbf{A})$ is unique if and only if the directed graph associated to $\mathbf{A}$ is weakly connected.

Proof. See Bozóki et al. [2, Theorem 2].

Theorem 3. EM may violate LOP.

Proof. Consider the directed acyclic graph on Figure 3. An associated ordinal pairwise comparison matrix $\mathbf{A}$ is as follows:

$$
\mathbf{A}=\left(\begin{array}{cccccccc}
1 & 3 & * & * & * & * & 3 & 3 \\
1 / 3 & 1 & 3 & 3 & * & * & * & * \\
* & 1 / 3 & 1 & * & 3 & 3 & * & * \\
* & 1 / 3 & * & 1 & 3 & 3 & * & * \\
* & * & 1 / 3 & 1 / 3 & 1 & * & 3 & 3 \\
* & * & 1 / 3 & 1 / 3 & * & 1 & 3 & 3 \\
1 / 3 & * & * & * & 1 / 3 & 1 / 3 & 1 & * \\
1 / 3 & * & * & * & 1 / 3 & 1 / 3 & * & 1
\end{array}\right) .
$$

Property $L O P$ is satisfied if $w_{1}(\mathbf{A}) \geq w_{2}(\mathbf{A}), w_{1}(\mathbf{A}) \geq w_{7}(\mathbf{A}), w_{1}(\mathbf{A}) \geq w_{8}(\mathbf{A})$, $w_{2}(\mathbf{A}) \geq w_{3}(\mathbf{A}), w_{2}(\mathbf{A}) \geq w_{4}(\mathbf{A})$ as well as $w_{i}(\mathbf{A}) \geq w_{j}(\mathbf{A})$ for all $i=3,4$ and $j=5,6$; $i=5,6$ and $j=7,8$.

However, $E M$ results in

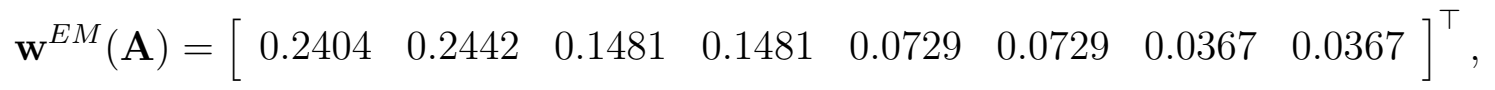

that is, $w_{1}^{E M}(\mathbf{A})<w_{2}^{E M}(\mathbf{A})$, in contradiction with preservation of linear order.

A parallel of Corollary 1 is not true in the case of $E M$, it may give a different ranking for a certain ordinal pairwise comparison matrix corresponding to the same directed acyclic graph.

Proposition 3. Let $\mathbf{A}=\left[a_{i j}\right]$ be an ordinal pairwise comparison matrix representing a directed acyclic graph. The ranking of the alternatives according to EM depends on the value of $b>1$.

Proof. Consider the directed acyclic graph on Figure 3, Besides A, another representation by ordinal pairwise comparison matrix $\mathbf{A}^{\prime}$ is as follows:

$$
\mathbf{A}^{\prime}=\left(\begin{array}{cccccccc}
1 & 4 & * & * & * & * & 4 & 4 \\
1 / 4 & 1 & 4 & 4 & * & * & * & * \\
* & 1 / 4 & 1 & * & 4 & 4 & * & * \\
* & 1 / 4 & * & 1 & 4 & 4 & * & * \\
* & * & 1 / 4 & 1 / 4 & 1 & * & 4 & 4 \\
* & * & 1 / 4 & 1 / 4 & * & 1 & 4 & 4 \\
1 / 4 & * & * & * & 1 / 4 & 1 / 4 & 1 & * \\
1 / 4 & * & * & * & 1 / 4 & 1 / 4 & * & 1
\end{array}\right) .
$$


$E M$ gives

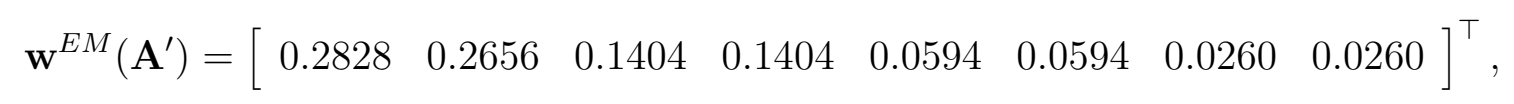

thus $w_{1}^{E M}(\mathbf{A})<w_{2}^{E M}(\mathbf{A})$ but $w_{1}^{E M}\left(\mathbf{A}^{\prime}\right)>w_{2}^{E M}\left(\mathbf{A}^{\prime}\right)$, which verifies Proposition 3 ,

Note that Proposition 3 does not necessarily mean the violation of linear order preservation if the $a_{i j}$ is missing. For instance, the relative ranking of alternatives 6 and 7 can be arbitrary in Example 2.

Remark 4. There exists an example with 6 alternatives and 5 known comparisons (which is minimal provided weak connectedness) demonstrating Proposition 3, It is the smallest in the number of alternatives.

The sensitivity of the ranking of the alternatives by $E M$ to the choice of $b>1$ was observed by Genest et al. [6] for certain complete pairwise comparison matrices.

\section{Conclusion}

Logarithmic Least Squares Method seems to give a counter-intuitive ranking of the alternatives for some incomplete pairwise comparison matrices representing preferences described by a directed acyclic graph. The ranking according to the Eigenvector Method may also contradict to the natural ranking order, while it depends on the correspondence chosen for these preferences, too.

Our results open at least three topics for future research:

1. How can one characterize the set of ordinal pairwise comparison matrices with a linear order of the alternatives for which $L L S M$ obeys $L O P$.

2. When does an unambiguous ranking of the alternatives according to $E M$ exist (on the class of preferences given by a directed acyclic graph)?

3. Which weighting methods perform well with respect to the condition $L O P$ ?

\section{References}

[1] Bana e Costa, C. A. and Vansnick, J.-C. (2008). A critical analysis of the eigenvalue method used to derive priorities in AHP. European Journal of Operational Research, 187(3):1422-1428.

[2] Bozóki, S., Fülöp, J., and Rónyai, L. (2010). On optimal completion of incomplete pairwise comparison matrices. Mathematical and Computer Modelling, 52(1-2):318-333.

[3] Crawford, G. and Williams, C. (1980). Analysis of subjective judgment matrices. Interim report R-2572-AF, Rand Corporation, Santa Monica.

[4] Crawford, G. and Williams, C. (1985). A note on the analysis of subjective judgment matrices. Journal of Mathematical Psychology, 29(4):387-405.

\footnotetext{
${ }^{4}$ One may realize that all counterexamples have a certain structure, visible on the family of directed acyclic graphs according to Example 4 the first alternative is preferred to the second and some with a low rank, while the second is preferred to some others with a high rank.
} 
[5] De Graan, J. G. (1980). Extensions of the multiple criteria analysis method of T. L. Saaty. Voorburg. National Institute for Water Supply.

[6] Genest, C., Lapointe, F., and Drury, S. W. (1993). On a proposal of Jensen for the analysis of ordinal pairwise preferences using Saaty's eigenvector scaling method. Journal of Mathematical Psychology, 37(4):575-610.

[7] Harker, P. T. (1987). Incomplete pairwise comparisons in the analytic hierarchy process. Mathematical Modelling, 9(11):837-848.

[8] Jensen, R. E. (1986). Comparison of consensus methods for priority ranking problems. Decision Sciences, 17(2):195-211.

[9] Kaiser, H. F. and Serlin, R. C. (1978). Contributions to the method of paired comparisons. Applied Psychological Measurement, 2(3):423-432.

[10] Kwiesielewicz, M. (1996). The logarithmic least squares and the generalized pseudoinverse in estimating ratios. European Journal of Operational Research, 93(3):611-619.

[11] Perron, O. (1907). Zur Theorie der Matrices. Mathematische Annalen, 64(2):248-263.

[12] Saaty, T. L. (1980). The Analytic Hierarchy Process: planning, priority setting, resource allocation. McGraw-Hill, New York.

[13] Shiraishi, S. and Obata, T. (2002). On a maximization problem arising from a positive reciprocal matrix in ahp. Bulletin of informatics and cybernetics, 34(2):91-96.

[14] Shiraishi, S., Obata, T., and Daigo, M. (1998). Properties of a positive reciprocal matrix and their application to AHP. Journal of the Operations Research Society of Japan-Keiei Kagaku, 41(3):404-414. 